'Department of Cardiology, West German Heart Center Essen, University of Duisburg-Essen, Germany.

\title{
Mechanism of Coronary Malperfusion Due to Type-A Aortic Dissection
}

\author{
Rolf Alexander Jánosi, Thomas Buck, Raimund Erbel ${ }^{1}$
}

Herz 2009;34:478

DOI 10.1007/

s00059-009-3272-z
A 44-year-old man was admitted to a community hospital with acute onset of chest pain.

Based on ECG findings and a mild troponin elevation, the patient was initially treated for acute coronary syndrome. On the 2nd day of hospitalization,
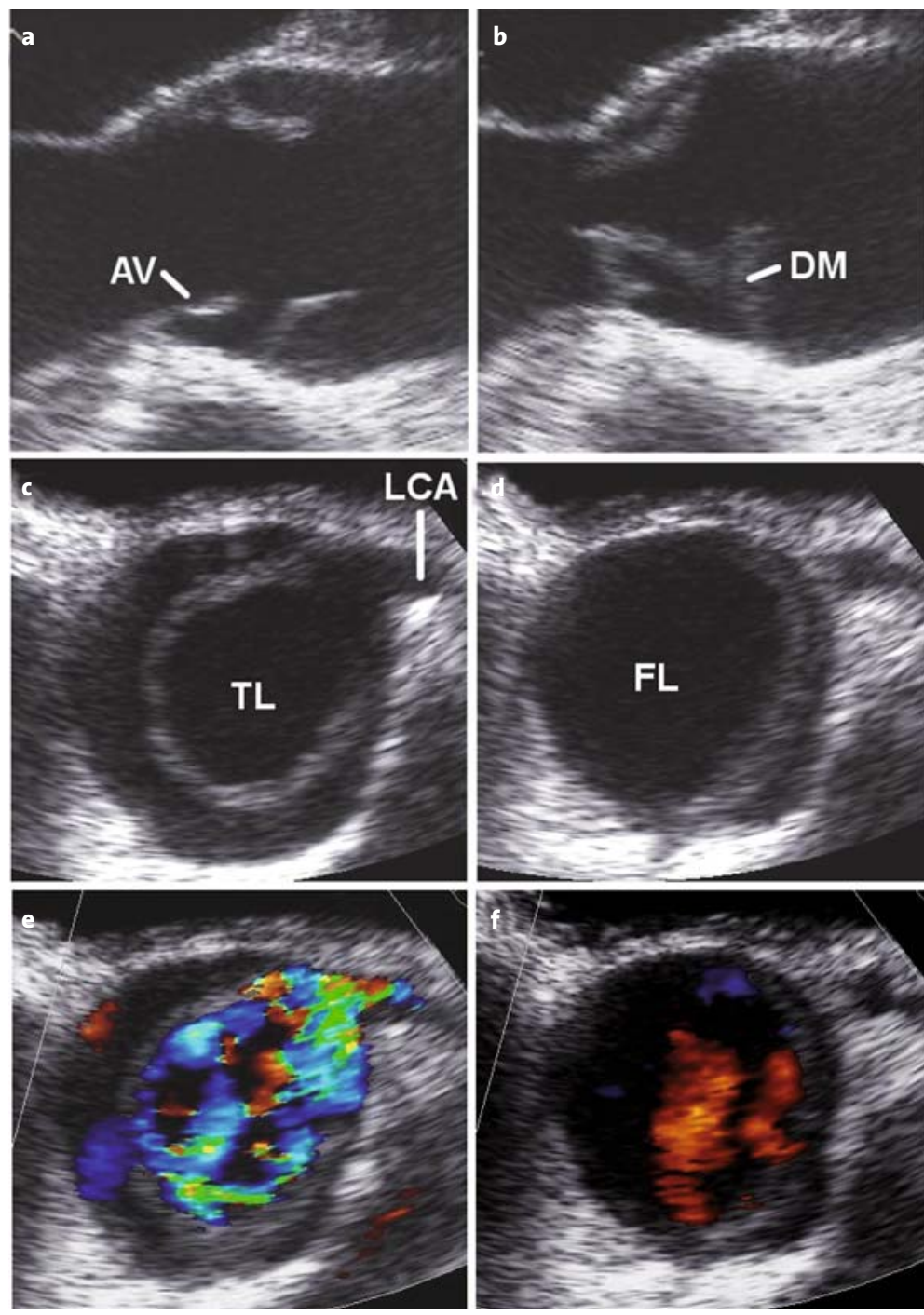

Figures $\mathbf{1 a}$ to 1f. Systolic (a, c, e) and diastolic (b, d, f) movement of the dissection membrane (DM) with prolapse of the dissection membrane through the aortic valve (AV). Short-axis view of the aortic valve showing the intermittent obstruction of the left coronary ostia (c-f; LCA). FL: false lumen; TL: true lumen. troponin increased to $12 \mathrm{ng} / \mathrm{ml}$ and transthoracic echocardiography showed severe aortic regurgitation, so that computed tomography was performed and revealed Stanford type-A aortic dissection of the ascending aorta. The patient was immediately transferred to the hybrid operating room of our hospital.

Preoperative coronary angiography showed nonstenotic coronary heart disease. Intraoperative transesophageal echocardiography revealed an intimal flap beginning just above the aortic valve. During diastole, the intimal flap prolapsed through the aortic valve, resulting in intermittent diastolic occlusion of the coronary ostia (Figure 1). Emergency operation resulted in successful replacement of the ascending aorta and reconstruction of the aortic valve.

The postoperative course was uneventful.

Aortic dissection coexisting with coronary malperfusion is a relatively rare $[1,2]$ but fatal condition. Even in the absence of obstructive coronary artery disease, these patients can suffer from significant impairment of myocardial perfusion due to diastolic collapse of the true lumen and occlusion of the coronary ostia by the dissection membrane. Ultimately, symptoms can mimic acute myocardial infarction to the point that thrombolytic treatment may be initiated inappropriately.

\section{References}

1. Neri E, Toscano T, Papalia U, et al. Proximal aortic dissection with coronary malperfusion: presentation, management, and outcome. J Thorac Cardiovasc Surg 2001;121:552-60.

2. Zotz R, Stern H, Mohr-Kahaly S, et al. Coronary insufficiency in type II aortic dissection. Z Kardiol 1987;76:784-6.

\section{Address for Correspondence}

Rolf Alexander Jánosi, MD

Department of Cardiology

West German Heart Center Essen

University of Duisburg-Essen

Hufelandstraße 55

45122 Essen

Germany

Phone (+49/201) 723-84894, Fax -5401

e-mail: alexander@janosi.de 